\title{
Fatores Associados à Obesidade e ao Padrão Andróide de Distribuição da Gordura Corporal em Mulheres Climatéricas
}

\author{
Factors Related to Obesity and Android Pattern of Body Fat \\ Distribution in Climacteric Women
}

\begin{abstract}
Diana Beatriz Filip Raskin, Aarão Mendes Pinto-Neto, Lúcia Helena Simões Costa Paiva Analisa Raskin, Edson Zangiacomi Martinez
\end{abstract}

\section{RESUM0}

Objetivos: descrever as caracteristicas de um grupo de mulheres climatéricas, conhecer a freqüência e os fatores associados à obesidade e ao padrão andróide de distribuição da gordura corporal.

Métodos: estudo observacional com 518 pacientes com idade entre 45 e 65 anos em um ambulatório de climatério. Foram considerados a idade, cor, status menopausal, tempo de menopausa, atividade fisica, tabagismo, dieta, etilismo, antecedentes pessoais e familiares de hipertensão, diabetes, doença cardiovascular, dislipidemia e obesidade. O indice de massa corpórea e a relação das medidas cintura/quadril foram variáveis dependentes. Na análise estatística utilizaram-se os testes de Wilcoxon, $\chi^{2}$ de Pearson, com nivel de significância de 5\%, $e$ análise múltipla por regressão logística.

Resultados: mais de dois terços das participantes eram não-obesas com perfil andróide $e$ menopausadas. Aproximadamente um quarto tinha atividade fisica adequada, era tabagista, metade referiu dieta inadequada e um quinto era etilista. Pacientes com perfil andróide apresentaram média etária maior que mulheres com padrão ginecóide. Antecedentes pessoais de obesidade, hipertensão, diabetes e história familiar de diabetes relacionaram-se com obesidade e padrão andróide. O status pós-menopausa associou-se significativamente ao perfil andróide.

Conclusões: a maioria das mulheres foram não-obesas com perfil andróide, brancas, pósmenopáusicas, sedentárias, não-tabagistas nem etilistas. Os principais fatores associados à obesidade e padrão andróide foram os antecedentes pessoais de obesidade, hipertensão arterial, diabetes, história familiar de diabetes e, particularmente, o status pós-menopausa com o perfil andróide.

PALAVRAS-CHAVE: Menopausa. Obesidade. Perfil andróide.

\section{Introdução}

As mudanças observadas no perfil biofísico feminino ao longo dos anos, e particularmente na

Trabalho realizado no Ambulatório de Climatério da Faculdade de Ciências Médicas da Pontifícia Universidade Católica de Campinas/Departamento de Tocoginecologia da Universidade Estadual de Campinas.

Correspondência:

Diana Filip Raskin

a/c - ASTEC/CAISM/UNICAMP

Rua Alexander Fleming, 101 - Cidade Universitária

"Zeferino Vaz"

13.023-230 - Campinas - SP

Fone: (19) 788-9402 - e-mail: dbfr@zipmail.com.br fase climatérica, resultam do ganho ponderal, do aumento da gordura corporal pela obesidade e de alterações na composição e distribuição do tecido adiposo $^{1,2}$. Essas modificações, muito além de considerações estéticas, associam-se a anormalidades metabólicas implicadas na gênese e progressão da doença vascular aterosclerótica ${ }^{3,4}$.

A obesidade, definida como um aumento do depósito de triglicérides nas células adiposas determinado por um desequilibrio entre consumo e gasto de energia ${ }^{5}$, é considerada uma doença complexa e multifatorial, de prevalência crescente, que já atingiu proporções epidêmicas, acometendo cerca de $30 \%$ das mulheres adultas na socie- 
dade ocidental ${ }^{6,7}$. A literatura reconhece sua relação direta ou indireta com aumento da incidência de doenças crônicas, como as moléstias cardiocirculatórias que se associam à hipertensão arterial, diabetes mellitus não-insulino-dependente e dislipidemias, as colecistopatias, esteatose hepática e artropatias degenerativas e sua influência no risco de câncer de mama, endométrio, vesícula biliar e cólon ${ }^{3,5,7}$.

$\mathrm{O}$ acúmulo de gordura visceral com distribuição central ou abdominal predominante, que caracteriza o perfil andróide e é encontrado em mulheres climatéricas e pós-menopáusicas, é metabolicamente diferente da periférica ou glúteofemural que define o padrão ginecóide, prevalente no período reprodutivo ${ }^{1}$. O predomínio de adipócitos viscerais de natureza lipolitica e as altas taxas de lipólise abdominal provocam o aumento do fluxo portal de ácidos graxos livres e concentrações séricas elevadas de triglicérides e LDL colesterol e reduzidas de HDL colesterol, que acontecem simultaneamente na vigência ou ausência de hipercolesterolemia ${ }^{8,9}$. Adicionalmente ocorre menor sintese de proteinas transportadoras de hormônios sexuais, aumento da testosterona livre e redução do clearance da insulina. Observamse ainda intolerância à glicose, hipercoagulabilidade e hipofibrinólise associadas à hipersecreção de insulina desencadeada pelos níveis de ácidos graxos circulantes, apesar do estado de maior resistência periférica ${ }^{2,3,9}$.

Estudos anteriores ${ }^{8,10,11}$ e publicações recentes ${ }^{4,7,9}$ destacaram a ocorrência de mudanças no padrão da gordura corporal, associadas ou independentes da obesidade, em função do próprio envelhecimento, das condições hormonais do climatério e pós-menopausa, tempo de menopausa ${ }^{1,2}$, de fatores genéticos, ambientais e comportamentais como sedentarismo, dietas com predomínio de gorduras saturadas, tabagismo e etilismo. Também reconheceram sua importância clínica, em virtude das complicações metabólicas resultantes, considerando a obesidade e o padrão andróide fatores de risco cardiovasculare ${ }^{12-15}$.

A magnitude deste problema, apontada pela prevalência crescente de obesidade, ocorrência de perfil andróide e suas relações com o status menopausal, motivou a realização deste estudo com objetivo de conhecer as características sociodemográficas, a freqüência de obesidade e perfil andróide e os fatores associados à sua presença em um grupo de mulheres climatéricas.

\section{Pacientes e Métodos}

Realizou-se estudo observacional de corte transversal com informações obtidas de 518 prontuários de mulheres assistidas no Ambulatório de Climatério da Faculdade de Ciências Médicas da Pontificia Universidade Católica, PUC, Campinas, entre 1 de outubro de 1997 e 15 de outubro de 1998. O presente estudo recebeu aprovação do Comitê de Ética em Pesquisa da Faculdade de Ciências Médicas, PUC, Campinas.

Foram incluídas no estudo as fichas clínicas de pacientes com idade entre 45 e 65 anos, com dados antropométricos (peso, altura e circunferências da cintura e quadril) e dosagem plasmática de FSH, quando necessária, para confirmar o estado menopausal.

Foram excluídas as portadoras de diabetes mellitus e ou dislipidemia em uso de hipoglicemiantes orais ou insulina e hipolipemiantes, respectivamente. As pacientes com antecedentes e ou doenças da tiróide, confirmados por exames laboratoriais, em tratamento ou não e as usuárias de terapia de reposição ou contraceptivos hormonais, por período igual ou superior a 180 dias nos seis meses anteriores à consulta. Também, as que estavam utilizando ou tinham utilizado diuréticos tiazídicos e ou beta-bloqueadores nos 3 meses anteriores à consulta.

No estudo das variáveis independentes e dependentes, classificadas em duas categorias, consideraram-se os auto-relatos, informações e dados numéricos anotados nos prontuários. As variáveis independentes foram a idade, a cor da pele, e o status menopausal. Este foi categorizado em perimenopausa, caracterizada pela presença referida de sangramento menstrual irregular após os 40 anos, e pós-menopausa, pela ausência de menstruação por período igual ou superior a 12 meses consecutivos ou pela dosagem plasmática de $\mathrm{FSH} \geq 40 \mathrm{mUI} / \mathrm{mL}$, e o tempo de menopausa. A atividade fisica regular foi caracterizada por referências à prática, freqüência semanal e duração diária de caminhadas, ginástica e/ou hidroginástica, por periodo igual ou superior a seis meses no último ano. Foi considerada adequada quando realizada em número igual ou superior a três vezes com duração mínima de 45 minutos $^{13}$. O tabagismo foi definido como o hábito de fumar, independente do número e tipo de cigarros consumidos por dia, nos últimos cinco anos ${ }^{11}$. O tipo de dieta foi caracterizado pela avaliação qualitativa e freqüência semanal relativas ao consumo de gorduras insaturadas e ingestão de cálcio, no último ano. Considerando-se as carnes brancas e magras, laticinios desnatados, queijos brancos, cereais, grãos, fibras, vegetais e frutas, foi classificada como adequada, quando referida com freqüência semanal igual ou superior a três vezes ${ }^{14}$. O etilismo foi definido como consumo regular de be- 
bidas alcóolicas nos 12 meses precedentes. Foi considerado presente quando o consumo foi igual ou superior a 30 gramas/álcool/dia, correspondendo a duas garrafas de cerveja ou três copos de vinho ou duas doses de bebida destilada ou aguardente e estimado pelo conteúdo alcóolico de cada tipo. Foi classificado como ausente, quando o consumo era inferior a 30 gramas/álcool/dia ${ }^{15}$. Foram anotados os antecedentes mórbidos pessoais e familiares, considerando-se a ausência ou presença relativas a atendimentos e exames médicos, diagnósticos clínicos e/ou laboratoriais, internações hospitalares, tratamentos realizados e/ou vigentes. Foram especificamente investigados: hipertensão arterial, diabetes mellitus e doença cardiovascular, incluindo coronariopatia isquêmica definida, classificada como prematura se referida antes dos 55 anos, e/ou diagnóstico de doença vascular cerebral oclusiva. Os antecedentes pessoais de hipercolesterolemia, doença vascular periférica (varizes em membros inferiores, tromboflebites e/ou doença arterial oclusiva), e de obesidade. Esta última foi caracterizada como ganho ponderal igual ou superior a $10 \mathrm{~kg}$ nos últimos dez anos ou após os 35 anos. Também foi considerada a história familiar de doença cardiovascular e de dislipidemia.

A variável dependente foi o índice de massa corpórea, medida definida pelo quociente dos valores do peso em quilogramas e do quadrado da altura em metros ${ }^{16}$. Classificaram-se as pacientes em não-obesas, quando esse índice foi menor que $30 \mathrm{~kg} / \mathrm{m}^{2}$, e obesas, se igual ou superior a $30 \mathrm{~kg} / \mathrm{m}^{2}{ }^{16}$. O padrão de distribuição da gordura corporal, definido pela relação das medidas das circunferências da cintura e quadril, foi categorizado pelo valor dessa razão em ginecóide, periférico ou glúteo-femural, quando menor ou igual a 0,80 , e andróide, central ou abdominal, se maior que $0,80^{8,10}$. Considerou-se o valor numérico de uma única medida de peso, altura e das circunferências nos cálculos dos índices de massa corpórea e cintura/quadril.

$\mathrm{Na}$ análise estatística aplicaram-se os testes não-paramétrico de Wilcoxon unilateral na avaliação das médias e desvio-padrão da idade e do tempo de menopausa, e o $\chi^{2}$ de Pearson, para análise das tabelas de freqüencia e das relações entre variáveis independentes e dependentes, observando-se um nível de significância de $5 \%$. Finalmente procedeu-se à análise por modelo de regressão logística com variáveis múltiplas e processo de seleção stepwise incluindo todas as variáveis estudadas, exceto a idade e o tempo de menopausa, não analisados neste modelo por apresentarem colinearidade com status pós-menopau$\mathrm{sa}^{17}$.

\section{Resultados}

A análise das características deste grupo revelou predominio de mulheres brancas, na pósmenopausa, não-obesas com padrão andróide. Destacaram-se as participantes com atividade fisica e dieta inadequadas, não-fumantes e nãoetilistas (Tabela 1). A média de idade das participantes deste estudo foi de $51,7 \pm 5,1$ anos. A média etária das pacientes não-obesas e obesas foi semelhante. Entretanto, notou-se que mulheres com perfil andróide apresentaram média de idade maior que as portadoras de padrão ginecóide (Tabela 2).

Tabela 1 - Distribuição porcentual das mulheres segundo características, estilo de vida e hábitos $(\mathrm{n}=518)$

\begin{tabular}{llcc}
\hline Características & & $\mathbf{n}$ & $\%$ \\
\hline Idade & $\geq 51$ anos & 269 & 51,9 \\
Cor & Branca & 354 & 68,3 \\
Status menopausal & Pós-menopausa & 337 & 65,0 \\
Tempo de menopausa * & $\geq 4$ anos & 169 & 50,1 \\
Obesas & IMC $\geq 30 \mathrm{~kg} / \mathrm{m}^{2}$ & 176 & 34,0 \\
Padrão andróide & $\mathrm{RCQ}>0,80$ & 401 & 77,4 \\
Atividade física regular & Inadequada & 390 & 75,3 \\
Dieta & Inadequada & 290 & 56,0 \\
Etilismo & Ausente & 416 & 80,3 \\
\hline
\end{tabular}

${ }^{*} n=337 ; \mathrm{IMC}=$ índice de massa corpórea; $R C Q$ = relação cintura/quadril

Tabela 2- Características das mulheres segundo índice de massa corpórea, padrão de distribuição da gordura e média etária em anos.

\begin{tabular}{lcccc}
\hline Características & Média etária & Desvio-padrão & $\mathbf{p}^{*}$ & $\mathbf{n}$ \\
\hline Não-obesas ** & 51,6 & 5,33 & NS & 342 \\
Obesas ** $^{*}$ & 52,0 & 4,61 & & 176 \\
Ginecóide ** $^{*}$ & 48,8 & 3,63 & $<0,01$ & 117 \\
Andróide ** $^{*}$ & 52,6 & 5,15 & & 401
\end{tabular}

${ }^{*}$ Teste de Wilcoxon unilateral; **|dade: mínima 45 anos - máxima 65 anos, NS: não significativo

Ao se considerar a distribuição das mulheres segundo idade, cor, status, tempo de menopausa, padrão de distribuição da gordura, hábitos e indice de massa corpórea verificou-se porcentual maior de participantes com idade inferior a 51 anos $(70,3 \%)$, brancas $(68,4 \%)$, na perimenopausa $(71,3 \%)$, com perfil ginecóide $(81,2 \%)$ e fumantes $(76,2 \%)$ entre as mulheres não-obesas, e predomínio do padrão andróide entre as obesas. Quanto aos hábitos relatados, observou-se que o número 
de pacientes não-obesas fumantes foi três vezes maior que o de obesas, mostrando associação significativa entre tabagismo e índice de massa corpórea inferior a $30 \mathrm{~kg} / \mathrm{m}^{2}(\mathrm{p}<0,01)$. Com relação aos antecedentes pessoais, destacaram-se pela maior ocorrência os de obesidade $(64,1 \%)$, diabetes $(46,5 \%)$ e hipertensão arterial (46\%). Dentre os familiares, referências de hipertensão arterial e diabetes foram os mais freqüentes nas mulheres obesas (tabelas não-apresentadas).

A análise por modelo de regressão logistica identificou os antecedentes pessoais de obesidade e hipertensão arterial e a história familiar de diabetes como variáveis significativamente associadas ao índice de massa corpórea igual ou superior a $30 \mathrm{~kg} / \mathrm{m}^{2}$ (Tabela 3 ).

Tabela 3 - Variáveis significativamente associadas à obesidade $(n=518)$.

\begin{tabular}{lcccc}
\hline Variáveis & Coeficiente & EP & $\begin{array}{c}\text { Odds } \\
\text { ratio }\end{array}$ & $\mathbf{p}$ \\
\hline Constante & $-1,8305$ & 0,1826 & - & $<0,01$ \\
AP obesidade & 1,7085 & 0,2203 & 5,521 & $<0,01$ \\
AP hipertensão arterial & 0,8415 & 0,2073 & 2,320 & $<0,01$ \\
AF diabetes & 0,6470 & 0,2129 & 1,910 & $<0,01$ \\
\hline
\end{tabular}

EP: erro-padrão do coeficiente. AP: antecedentes pessoais. AF: antecedentes familiares. Variáveis analisadas: cor, status menopausal, padrão de distribuição de gordura, estilo de vida, hábitos, antecedentes pessoais e familiares

Ao avaliar a distribuição das mulheres segundo idade, cor, status, tempo de menopausa, indice de massa corpórea, hábitos e padrão de distribuição da gordura verificou-se porcentual significativamente maior de participantes com idade igual ou superior a 51 anos, portadoras de padrão andróide $(89,2 \%)$. O número de pacientes perimenopáusicas com perfil ginecóide e padrão andróide foi semelhante. Entretanto, observamos predomínio do padrão andróide nas mulheres pósmenopausadas $(92,6 \%)$, nas obesas $(87,5 \%)$ e nas não-etilistas $(80 \%)(p<0,05)$. Também este perfil mostrou-se prevalente $(79,3 \%)$, mas não-significativo entre as não-fumantes $(\mathrm{p}=0,06)$. Quando considerados os antecedentes pessoais, familiares e o padrão de distribuição da gordura, notouse freqüência maior e significativa de referências pessoais de diabetes $(91,6 \%)$, obesidade $(89,4 \%)$, doença cardiovascular $(88,4 \%)$, hipercolesterolemia $(87,5 \%)$ e hipertensão arterial $(86,6 \%)$ nas portadoras de distribuição central da gordura, relacionando-se diretamente com o perfil andróide $(p<0,05)$ (tabelas não-apresentadas).

A análise por modelo de regressão logística identificou o status pós-menopausa e os antece- dentes pessoais de diabetes, hipertensão arterial e obesidade como variáveis significativamente associadas ao padrão andróide (Tabela 4).

Tabela 4 - Variáveis significativamente associadas ao padrão andróide $(n=518)$.

\begin{tabular}{lcccr}
\hline Variáveis & Coeficiente & EP & $\begin{array}{c}\text { Odds } \\
\text { ratio }\end{array}$ & p \\
\hline Constante & $-3,1144$ & 0,4017 & - & $<0,01$ \\
Pós-menopausa & 2,5188 & 0,2639 & 12,414 & $<0,01$ \\
AP diabetes & 0,9970 & 0,4892 & 2,710 & 0,04 \\
AP hipertensão arterial & 0,8595 & 0,2713 & 2,362 & $<0,01$ \\
AP obesidade & 0,8318 & 0,3350 & 2,297 & 0,01 \\
\hline
\end{tabular}

EP: erro-padrão do coeficiente. AP: antecedentes pessoais. AF: antecedentes familiares. Variáveis analisadas: cor, status menopausal, índice de massa corpórea, estilo de vida, hábitos, antecedentes pessoais e familiares.

\section{Discussão}

O presente estudo constatou uma freqüência de obesidade condizente com a prevalência de obesidade estimada em pesquisas populacionais recentes ${ }^{6,18}$. Mostrou peculiaridades caracterizadas pelo predomínio de pacientes pós-menopáusicas, sedentárias, com dieta considerada inadequada, assim como relação entre obesidade e distribuição central da gordura corporal, que poderiam explicar a maior freqüência de portadoras de perfil andróide $(77,4 \%)$, quando comparada às encontradas em estudos populacionais, que variaram de 25 a 48\% ${ }^{18-20}$. Confirmou a importância dos antecedentes pessoais de obesidade, hipertensão arterial, diabetes e história familiar de diabetes como fatores relacionados à obesidade e perfil andróide, destacando o papel do status pós-menopausa na distribuição central da gordura.

Ao analisar o estilo de vida e tipo de dieta, observamos que os porcentuais de pacientes sedentárias e com dieta inadequada ( 75 e $56 \%$ dos casos) foram superiores aos descritos $(70,5 \mathrm{e}$ $52,2 \%)$ em estudo populacional realizado em nosso meio ${ }^{21}$ e diferentes dos registrados em pesquisas americanas que apontaram ocorrências menor de atividade fisica inadequada e maior de hábitos alimentares adversos ${ }^{13,14,22}$.

Verificou-se que $24 \%$ das participantes eram tabagistas e $20 \%$ etilistas. Esses resultados foram semelhantes aos encontrados em mulheres americanas e em estudo populacional desenvolvido no interior paulista ${ }^{11,21}$. Porém, foram inferiores aos constatados em outras investigações, que mostraram valores entre 25 e $60 \%{ }^{18,20}$. Entretanto, pesquisa populacional gaúcha mostrou que $4,2 \%$ das 
mulheres (com média etária de 43,2 anos) referiram o etilismo como hábito ${ }^{23}$.

A média etária significativamente maior das participantes e características sociodemográficas e culturais poderiam explicar a maior freqüência de hábitos adversos como o sedentarismo e dieta inadequada. É oportuno lembrar que estas características comportamentais e suas mudanças sofrem influências regionais, dependem de integração social e familiar e de oportunidades de acesso aos serviços de saúde, particularmente nos grupos menos favorecidos.

Verificamos que a média de idade das pacientes não-obesas e obesas foi semelhante, em concordância com os resultados de estudos prospectivo e de caso-controle realizados com holandesas e italianas pós-menopausadas ${ }^{18,24}$. Contrariamente, evidências epidemiológicas americanas e dados de estudo populacional nacional revelaram média etária significativamente maior em mulheres obesas, quando comparada com a das não-obesas ${ }^{23,25}$.

Os resultados deste trabalho, assim como os de outras pesquisas, não evidenciaram relações entre hábitos adversos e índice de massa corpórea, exceção feita ao hábito de fumar ${ }^{18,24,25}$.

A análise dos antecedentes pessoais e familiares mostrou a presença de obesidade relacionada às principais características de risco cardiovascular, sugerindo ainda, a influência de fatores genéticos na gênese da hipertensão e, principalmente, nas alterações do metabolismo dos carboidratos que se associaram à obesidade e padrão andróide. Outras pesquisas identificaram relações semelhantes ${ }^{4,23,26}$.

Sabe-se que as necessidades energéticas diminuem progressivamente, ao longo dos anos, e modificações físicas, caracterizadas pela perda de massa muscular e ganho de tecido adiposo, são observadas na mulher climatérica ${ }^{1,10,25}$. Destacamos a importância da idade na distribuição da gordura, caracterizada pelo perfil andróide predominante $(89,2 \%)$ entre mulheres com idade igual ou superior a 51 anos, associado ou não à obesidade. Destacamos também, a influência do status menopausal na adiposidade visceral abdominal, verificada em $82,6 \%$ das pós-menopausadas. O aumento da freqüência do padrão andróide com a maior idade das pacientes, e ainda sua presença marcante associada à pós-menopausa, foram descritos, respectivamente, em pesquisa populacional brasileira e em estudos observacionais e de caso controle com mulheres entre 45 e $69 \operatorname{anos}^{1,24,27}$.

Entretanto, o porcentual de pacientes perimenopáusicas $(49,2 \%)$ que, apesar do status menopausal, apresentaram perfil andróide sugere modificações na composição e distribuição da gordura, prédeterminadas pelo perfil hormonal androgênico, acontecendo provavelmente antes dos 45 anos ou associadas à obesidade, mostrando relações entre índice de massa corpórea e padrão central.

Cabe considerar ainda que o padrão andróide, predominante entre as participantes com antecedentes de hipertensão arterial, diabetes, doença cardiovascular, hipercolesterolemia e história familiar de dislipidemia, reflete mudanças biofísicas associadas a alterações pressóricas e metabólicas lipoprotéicas e glicêmicas, e traduzem a existência de um estado aterogênico, transformando este perfil em fator de risco coronariano ${ }^{8,9}$.

A associação inversa entre etilismo e perfil andróide identificada neste trabalho, atribuída à relação entre consumo exagerado de bebidas alcoólicas e concentrações plasmáticas elevadas de estrogênios, foi descrita em estudo observacional e ensaio clínico randomizado norte-america$\operatorname{nos}^{15,28}$. Acredita-se que o nível de estrogênio circulante seja maior nas mulheres que consomem grandes quantidades de bebidas alcóolicas do que nas etilistas moderadas e não-etilistas, devido à depuração metabólica reduzida ou ao aumento da secreção estrogênica determinada pelas concentrações plasmáticas elevadas de álcool.

Cabe considerar que as principais características que se relacionaram à obesidade e ao perfil andróide não foram precisamente as mesmas. Os dados sugerem a presença de entidades diferentes influenciando estes dois fatores. Sabe-se que se associam à doença cardiovascular e diabetes mellitus não insulino-dependente, sendo provavelmente as portadoras de padrão andróide as de maior risco coronariano ${ }^{25,29}$.

As diferenças observadas entre este e outros estudos devem-se possivelmente à natureza do mesmo, aos critérios adotados nos processos de seleção, à faixa etária e hábitos das participantes. O método aplicado na análise do perfil, independente do índice de massa corpórea, não foi o utilizado na maioria das pesquisas que geralmente consideraram a obesidade associada ao padrão de distribuição da gordura.

Por ser estudo hospitalar que avaliou um grupo selecionado de participantes, recomendamos cautela na interpretação dos resultados. Reconhecemos que estes não são extrapoláveis para a população, porém espera-se que sirvam como referência, pois representam a condição de um número considerável de mulheres que recebem assistência médica e de muitas outras que não procuram os serviços de saúde.

Finalmente, é oportuno lembrar que este trabalho, realizado com o intuito de conhecer o 
perfil das pacientes climatéricas, mostrou freqüências elevadas de obesidade e perfil andróide e caracterizou estilo de vida e hábitos, de certa forma, diferentes dos observados em outras populações, que alertam para o risco metabólico das participantes.

Estas observações enfatizam a prioridade de atitudes médicas e ações multidisciplinares concretas e coordenadas numa abordagem racional e global da mulher. Reforçam a necessidade de avaliação sistemática para identificar os fatores predisponentes e as pacientes de risco. Orientação nas mudanças de estilo de vida e hábitos, por meio de atividades informativas e educativas, e o tratamento criterioso, quando indicado, são indispensáveis para conseguir a redução, manutenção do peso e o controle adequado do índice cintura/ quadril, tendo por fim último a prevenção da doença cardiovascular.

\section{SUMMARY}

Purpose: to describe sociodemographic characteristics of a group of climacteric women in order to discover the frequency and the variables associated with obesity and android profile of body fat distribution.

Methods: an observational study was carried out in 518 patients aged 45 to 65 years, in a climacterium outpatient clinic. Age, color, menopausal status, duration of menopause, physical activity, smoking status, diet, alcohol intake, personal and family antecedents of arterial hypertension, diabetes mellitus, cardiovascular disease, dyslipidemia and obesity were considered. Body mass index and the waist/hip ratio were the dependent variables. For the statistical analysis Wilcoxon test, Pearson's correlation coefficient, with a $5 \%$ level of significance, and multivariate analysis using regression model were used.

Results: more than two thirds of the participants were nonobese with an android profile and postmenopausal. One fourth had physical activity and were smokers; half reported an inadequate diet and one fifth were alcoholics. Patients with an android profile presented higher mean age than women with gynecoid pattern. Personal antecedents of obesity, arterial hypertension, diabetes and family history of diabetes were related to obesity and android pattern. Postmenopausal status was significantly associated with the android profile.

Conclusions: the majority of the participants were nonobese with an android profile, white, postmenopausal, sedentary, neither smokers nor alcoholics. The main factors related to obesity and android pattern were personal antecedents of obesity, arterial hypertension, diabetes, family history of diabetes and particularly, postmenopausal status with android profile.

KEY WORDS: Menopause. Obesity. Android profile.

\section{Referências}

1. Tremollières FA, Pouilles JM, Ribot CA. Relative influence of age and menopause on total and regional body composition changes in postmenopausal women. Am J Obstet Gynecol 1996; 175:1594-600.

2. Turcato E, Zamboni M, De Pergola G, et al. Interrelationships between weight loss, body fat distribution and sex hormones in pre- and postmenopausal obese women. J Intern Med 1997; 241:363-72.

3. Björntorp P. Body fat distribution, insulin resistance, and metabolic diseases. Nutrition 1997; 13:795803.

4. Jousilahti P, Vartiainen E, Tuomilehto J, Puska P. Sex, age, cardiovascular risk factors, and coronary heart disease: a prospective follow-up study of 14.786 middle-aged men and women in Finland. Circulation 1999; 99:1165-72.

5. Must A, Spadano J, Coakley EH, Field AE, Colditz G, Dietz WH. The disease burden associated with overweight and obesity. JAMA 1999; 282:523-9.

6. Mokdad AH, Serdula MK, Dietz WH, Bowman BA, Marks JS, Koplan JP. The spread of the obesity epidemic in the United States, 1991-1998. JAMA 1999; 282:1519-22.

7. Willett WC, Dietz WH, Colditz GA. Guidelines for healthy weight. N Engl J Med 1999; 341:427-34.

8. Pouliot MC, Desprès JP, Lemieux S, et al. Waist circumference and abdominal sagittal diameter: best simple anthropometric indexes of abdominal visceral adipose tissue accumulation and related cardiovascular risk in men and women. Am J Cardiol 1994; 73:460-8.

9. Couillard C, Lamarche B, Tchernof A, et al. Plasma high-density lipoprotein cholesterol but not apolipoprotein A-I is a good correlate of the visceral obesity-insulin resistance dyslipidemic syndrome. Metabolism 1996; 45:882-8.

10.Poehlman ET, Toth MJ, Gardner AW. Changes in energy balance and body composition at menopause: a controlled longitudinal study. Ann Intern Med 1995; 123:673-5.

11.Witteman JCM, Grobbee DE, Valkenburg HA, Hemert AM, Stijnen T, Hofman A. Cigarette smoking and the development and progression of aortic atherosclerosis. A 9-year population-based followup study in women. Circulation 1993; 88:2156-62. 
12.Samaras K, Spector TD, Nguyen TV, Baan K, Campbell LV, Kelly PJ. Independent genetic factors determine the amount and distribution of fat in women after the menopause. J Clin Endocrinol Metab 1997; 82:781-5.

13.Pate RR, Pratt M, Blair SN, et al. Physical activity and public health. A recommendation from the Centers Control and Prevention and the American College of Sports Medicine. JAMA 1995; 273:402-7.

14.Heini AF, Weinsier RL. Divergent trends in obesity and fat intake patterns: the American paradox. Am J Med 1997; 102:259-64.

15.Duncan BB, Chambless LE, Schmidt MI, et al. Association of the waist-to-hip ratio is different with wine than with beer or hard liquor consumption. Atherosclerosis Risk in Communities Study Investigators. Am J Epidemiol 1995; 142:1034-8.

16. Bray GA. Classification and evaluation of the obesities. Med Clin North Am 1989; 73:161-84.

17.Armitage P. Statistical methods in medical research. $3^{\text {rd }}$ ed. New York: Wiley J. \& Sons; 1974.

18.Seidell JC, Verschuren MM, van Leer EM, Kromhout D. Overweight, underweight, and mortality. A prospective study of 48.297 men and women. Arch Intern Med 1996; 156:958-63.

19.Lean ME, Han TS, Morrison CE. Waist circumference as a measure for indicating need for weight management. BMJ 1995; 311:158-61.

20.Kritz-Silverstein D, Barrett-Connor E. Long-term postmenopausal hormone use, obesity, and fat distribution in older women. JAMA 1996; 275:46-9.

21.Nicolau JC, Nogueira C, Maia LN, Ramores JAF. Evolução dos níveis de colesterol na população adulta de São José do Rio Preto (1991-1997). Arq Bras Cardiol 1998; 71:699-704.
22.Andersen R, Wadden TA, Bartlett SJ, Zemel B, Verde TJ, Franckowiak SC. Effects of lifestyle activity vs. structured aerobic exercise in obese women: a randomized trial. JAMA 1999; 281:335-40.

23.Gus M, Moreira LB, Pimentel M, Gleisener ALM, Moraes RJ, Fuchs FD. Associação entre diferentes indicadores de obesidade e prevalência de hipertensão arterial. Arq Bras Cardiol 1998; 70:111-4.

24.Gambacciani M, Ciaponi M, Cappagli B, et al. Body weight, body fat distribution, and hormonal replacement therapy in early postmenopausal women. J Clin Endocrinol Metab 1997; 82:414-7.

25.Rexrode KM, Hennekens $\mathrm{CH}$, Willett WC, et al. A prospective study of body mass index, weight change, and risk of stroke in women. JAMA 1997; 277:1539-45.

26.Willett WC, Manson JAE, Stampfer MJ, et al. Weight, weight change and coronary heart disease in women. Risk within the normal weight range. JAMA 1995; 273:461-5.

27.Guedes DP, Guedes JERP. Distribuição de gordura corporal, pressão arterial e níveis de lipídioslipoproteínas plasmáticas. Arq Bras Cardiol 1998; 70:93-8

28.Ginsburg ES, Walsh BW, Shea BF, Gao X, Gleason $\mathrm{RE}$, Barbieri RL. The effects of ethanol on the clearance of estradiol in postmenopausal women. Fertil Steril 1995; 63:1227-30.

29.Schmidt MI, Watson RL, Duncan BB, et al. Clustering of dyslipidemia, hyperuricemia, diabetes, and hypertension and its association with fasting insulin and central and overall obesity in a general population. Atherosclerosis Risk in Communities Study Investigators. Metabolism 1996; 45:699-706.

\section{RBGO}

\section{éumapublicaçãoda}

FEBRASGO

\section{equeaceitaartigos provenientes de ginecologistas,}

obstetrasedeoutras especialidades.

Portanto, publique!!!

Mande já seu artigo para RBGO 